\title{
The effects of ankle-foot orthoses with plantar flexion stop and plantar flexion resistance using rocker-sole shoes on stroke gait: A randomized-controlled trial
}

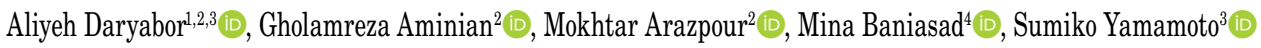 \\ 'Department of Physiotherapy, School of Rehabilitation, Shahid Beheshti University of Medical Sciences, Tehran, Iran \\ ${ }^{2}$ Department of Orthotics and Prosthetics, University of Social Welfare and Rehabilitation Sciences, Tehran, Iran \\ ${ }^{3}$ Department of Assistive Technological Science, Graduate School, International University of Health and Welfare, Japan \\ ${ }^{4}$ Department of Mechanical Engineering, Sharif University of Technology, Tehran, Iran
}

Received: May 19, 2020 Accepted: September 15, 2020 Published online: December 01, 2021

\begin{abstract}
Objectives: This study aims to evaluate the effect of two ankle-foot orthoses (AFOs), AFO with plantar flexion stop (AFO-PlfS), and AFO with plantar flexion resistance (AFO-PlfR), while wearing standard shoes and rocker-sole shoes.

Patients and methods: Between November 2017 and July 2018, in this randomized-controlled study, a total of 20 stroke patients ( 8 males, 12 females; mean age: 48.1 years; range, 33 to 65 years) in chronic phase were randomized to AFO groups (AFO-PlfS group, $n=10$ and AFO-PlfR group, $\mathrm{n}=10$ ). Each group received the allocated AFO along with two kinds of shoes (standard shoe and rocker shoe) for a two-week adaptation. Two effects were separately evaluated: The orthotic effect and rocker shoe effect were defined as the evaluation of using an AFO wearing standard shoe compared to only standard shoe, and evaluation of using an AFO wearing rocker shoe compared to an AFO wearing standard shoe, respectively. The gait of each group was measured by three-dimensional motion analysis.

Results: A significant orthotic effect was found in both AFO groups in spatiotemporal parameters and maximum ankle dorsiflexion in the single-support phase. Additionally, the AFO-PlfR group showed a significant improvement in the parameters related to the first rocker of gait, but not for AFO-PlfS group concerning the orthotic effect. The rocker shoe effect was found in significant reduction of peak ankle plantar flexor moment and power ankle generation during preswing for both AFO groups.

Conclusion: According to the orthotic effect, an AFO-PlfR can create better function in the improvement of parameters related to the first rocker. Although a rocker shoe can facilitate rollover for weight progression in the third rocker of gait, it cannot make a strong push-off function in stroke survivors.
\end{abstract}

Keywords: Gait, hemiplegia, orthotic devices, rocker shoe, stroke.

Hemiplegia damages to the ankle-foot complex and all function of gait rockers are disrupted, as described by Perry and Davids. ${ }^{[1]}$ In healthy individuals, weight transfer during initial-double support is helped by the heel rocker (first rocker) and during terminal double-support by the forefoot rocker (third rocker). ${ }^{[1]}$
Previous studies have shown that the greatest effects of hemiplegic gait impairments arise during double-support phases, while transferring weight from one limb to the next..$^{[2,3]}$

Among different rehabilitation approaches, wearing an ankle-foot orthosis (AFO) is clinically

Corresponding author: Gholamreza Aminian, PhD. Department of Orthotics and Prosthetics in the University of Social Welfare and Rehabilitation Sciences, Kodakyarst, Daneshjo Blvd., Evin, Tehran 1985713834, Iran.

e-mail: gholamrezaaminian@yahoo.com 
useful to improve stroke gait, and many studies have demonstrated the positive effects of their use. ${ }^{[4,5]}$ Among different AFOs categorized into non-articulated and articulated types, articulated AFOs can avoid drop foot successfully by generating dorsiflexion assisting force. ${ }^{[6,7]}$ The articulated AFOs with mechanical plantar flexion stops (AFO-PlfS) constrain other normal movement of the ankle probably, resulting in undesired knee flexion in early stance phase. ${ }^{[6,7]}$ On the other hand, findings of previous studies have shown that the plantar flexion resistive moment of AFOs (AFO-PlfR) plays a substantial role to gain heel contact in the heel rocker, and preserving the heel rocker is an important function for an AFO. ${ }^{[8-10]}$ Additionally, previous studies evaluating the mechanism of these AFOs on gait rockers of hemiplegia have reported that this mechanical AFO can improve the function of heel and ankle rockers to some extent, but not positive effect on forefoot rocker during push-off. ${ }^{[9,11,12]}$ The decreased ability to ankle push-off in patients suffering from central neurological disorders could be compensated by increased work of the hip joint. ${ }^{[13,14]}$ Moreover, a high energetic demand of gait is required resulting from this mechanical inefficiency. ${ }^{[15,16]}$ Since improving rollover and weight transfer in the third rocker is an essential function of the rocker-sole shoe (RSh) modification in prostheses and orthoses, it can be hypothesized that an forefoot rocker modification using an AFO is potentially able to improve rollover function of the ankle-foot complex and, as a result, facilitate weight progression from the paretic limb to the non-paretic one. ${ }^{[17,18]}$

A previous study reported that non-articulated rigid AFO modified with an RSh significantly increased hip extension and knee flexion at toe-off. ${ }^{[19]}$ It has been established that the moment or power produced by the plantar flexors should be considered as one of the most important determinants of push-off for stroke patients. ${ }^{[20,21]}$ However, these parameters were not evaluated in that study, ${ }^{[19]}$ and reported changes in hip and knee kinematics can be imagined as a mechanism to compensate the reduced ankle function in preswing phase. On the other hand, the rigid AFOs limit some movements having functional benefits. ${ }^{[6,7]}$ In the present study, we hypothesized that an AFO would have an orthotics effect by improving gait parameters in stroke patients, that gait parameters could be improved to a greater extent wearing the AFO-PlfR than the AFO-PlfS, and that the outcomes of push-off phase with use of RSh wearing an AFO would be better that use of standard shoe (SSh) wearing an AFO. We, therefore, aimed to compare the difference in gait with only SSh (without AFO) and with SSh on AFO as an orthotic effect, as well as gait with SSh on an AFO and with RSh on an AFO as a rocker shoe effect.

\section{PATIENTS AND METHODS}

This randomized-controlled study was conducted at University of Social Welfare and Rehabilitation Sciences, Department of Orthotics and Prosthetics, and Research Centre of Intelligent Neuro-Rehabilitation Technologies, between between November 2017 and July 2018. A total of 20 patients ( 8 males, 12 females; mean age: 48.1 years; range, 33 to 65 years) with hemiplegia secondary to stroke randomly classified under an AFO-PIfS group $(n=10)$ and an AFO-PlfR group $(n=10)$ were recruited. Inclusion criteria were as follows: age between 35 and 65 years; having a minimum of six months after stroke, a maximum spasticity score of 2 according to the Modified Ashworth Scale, and no use of a daily-wear AFO. Patients with deformities in their spine or lower limbs except for equinovarus of their injured limb resulting from hemiplegia, severe cardiorespiratory or cognitive problems, clawing toes, and proprioceptive sensory impairment were excluded from the study. Prior to study, all participants were informed about the nature of the study and a written informed consent was obtained. The study protocol was approved by the University of Social Welfare and Rehabilitation Science Ethics Committee (IR.USWR.REC.1395.399). The study was conducted in accordance with the principles of the Declaration of Helsinki.

\section{Orthotics and shoe fabrication}

The two kinds of custom-made AFOs with the mechanical ankle joints and plastic shell were provided by an expert orthotist for all patients. Since the patient wore AFOs within the shoes, a $1-\mathrm{cm}$ heel was put under patient's heel during casting to adapt with the footwear footplate. An AFO incorporating an overlap ankle joint in lateral and medial sides of affected limb was utilized as AFO-PlfS with stop to plantar flexion. An AFO-PlfR including an ankle joint with a spring in lateral side and single axis overlap joint in medial side was considered as the AFO with resistance to plantar flexion (Figure 1a). ${ }^{[22]}$ The ankle joint of this AFO generated a resistive moment, when the ankle joint moved to plantar flexion as specified in our previous study. ${ }^{[22]}$ The amount of the plantar flexion resistive moment could be tuned by adjusting a screw based on 
(a)

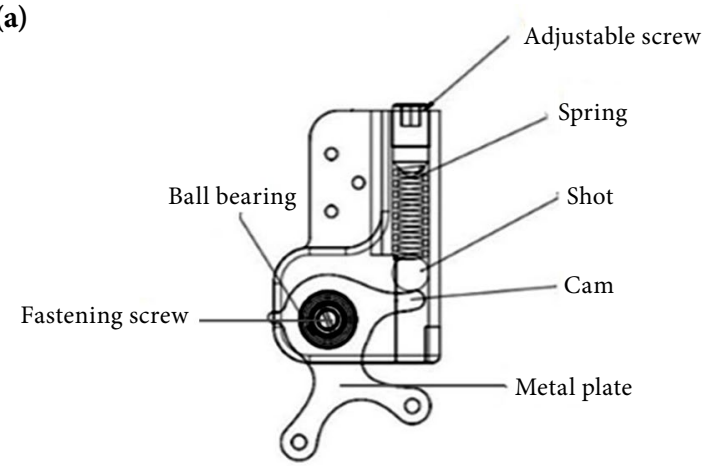

(b)

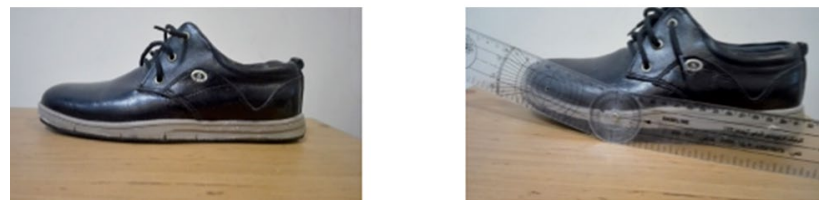

Figure 1. (a) A schematic diagram of the ankle joint of AFO that resists plantar flexion (AFO-PlfR), (b) standard shoe, (c) rocker shoe.

AFO-PlfR: Ankle-foot orthosis with plantar flexion resistance.

the patient's opinion and the orthotist's observation. The ankle joint of both AFOs allows free movement into dorsiflexion without resistance.

An initial fitting aligned with a gait training program was conducted by the orthotist and a physiotherapist. Also, when the AFO associated with the shoes was worn by the patients, the ankle was kept at $90^{\circ}$ to the lower leg using orthosis. Then, each participant received two shoe conditions with the allocated AFO. The main difference among these footwears was the design of the rocker sole. One pair had a mild rocker sole, which is the most basic, popular, and widely used for all shoes (SSh) (Figure 1b). The other pair was adapted with toe-only rocker sole, which has a significant rocker angle at the toe and only a mild rocker angle at the heel (Figure 1c). Therefore, a rocker modification was attached to the inferior portion of the SSh to provide RSh, which was made of ethylene-vinyl acetate rubber with standard hardness and $1-\mathrm{cm}$ thickness, and its angle was $15^{\circ}$ starting slightly from the proximal to the metatarsal heads (approximately $65 \%$ of the foot length relative to the back of the heel). The allocated AFO and footwears were used by the participants in outdoor for a two-week adaptation prior to quantitative gait testing. After two weeks, gait analyses were measured with patients' walking under three conditions in each group: walking with SSh, SSh on an AFO, and RSh on an AFO, with the order being randomly assigned.
A 10-Camera Vicon ${ }^{\circledR}$ digital motions capture system (Oxford, UK) and two force plates (Kistler, Switzerland and 9260AA6) were used for gathering data. A total of 49 reflective markers were tapped to the patients' landmarks in the Plug-in Gait full body modeling. The marker trajectories and the force plate data were measured at a sampling frequency of 120 and $1,200 \mathrm{~Hz}$, respectively. The measurements were repeated at patients' self-selected velocity, until three steps of their affected limb on a force plate and the healthy limb on another force plate were obtained.

\section{Outcome measures}

The Vicon's Nexus (Oxford, UK) version 2.6 software was used to compute the gait indices. The following parameters of the affected limb were analyzed in a gait cycle: spatiotemporal variables, peak value of the joint angles of the ankle, knee, and hip joints, internal ankle joint moment, hip abduction-adduction range of motion (ROM), three-dimensional (3D) ROM for pelvis and thorax, negative and positive peak powers of ankle, knee and hip joints, peak value of the posterior/anterior ground reaction force (GRF) component, and ROM of the vertical and lateral displacement of the center of mass (COM) in a gait cycle. We also analyzed vertical GRF for non-affected side to clarify whether an AFO wearing rocker shoe could decrease compensatory movement non-affected leg. In total, 38 gait indices were used for the analysis.

\section{Statistical analysis}

Considering the $95 \%$ confidence interval (CI) and $80 \%$ study power, gait variables for each group were recorded and averaged during three gait trials in each walking condition. Statistical analysis was performed using the IBM SPSS version 22.0 software (IBM Corp., Armonk, NY, USA). Descriptive data were expressed in mean \pm standard deviation (SD), median (interquartile range [IQR]) or number and frequency, where applicable. The Shapiro-Wilk test was conducted for the data normality. A repeated two-way analysis of variance (ANOVA) with paired factors (orthotic effect: only SSh and SSh on an AFO; rocker shoe effect: SSh on an AFO and RSh on an AFO) and an unpaired factor (AFO type) was applied for the normal distribution of data. The main effects of two factors were considered, when the interaction was not significant. When a significant interaction effect was found, a paired t-test analysis was performed for two conditions within each group. Non-parametric data were analyzed using 
the Wilcoxon signed-rank test for comparing the different conditions and the Mann-Whitney $U$ test for the AFO type. A $p$ value of $<0.05$ was considered statistically significant.

\section{RESULTS}

Table 1 shows the demographic characteristics of the patients. Totally, 10 stroke patients for each group were analyzed (Figure 2). In this study, the

\begin{tabular}{|c|c|c|c|c|c|}
\hline \multicolumn{6}{|c|}{$\begin{array}{c}\text { TABLE } 1 \\
\text { Clinical features of FM patients }\end{array}$} \\
\hline & \multicolumn{2}{|c|}{ AFO-PlfS $(n=10)$} & \multicolumn{2}{|c|}{ AFO-PlfR $(n=10)$} & \multirow[b]{2}{*}{$p$} \\
\hline & $\mathrm{n}$ & Mean \pm SD & $\mathrm{n}$ & Mean \pm SD & \\
\hline Age (year) & & $47.2 \pm 8.3$ & & $49 \pm 7.2$ & NS \\
\hline Sex & & & & & - \\
\hline Female & 6 & & 6 & & \\
\hline Male & 4 & & 4 & & \\
\hline Body height (m) & & $1.62 \pm 0.1$ & & $1.6 \pm 0.1$ & NS \\
\hline Body weight $(\mathrm{kg})$ & & $67.8 \pm 8.7$ & & $67.1 \pm 10.5$ & NS \\
\hline Paretic side & & & & & - \\
\hline Right & 5 & & 6 & & \\
\hline Left & 5 & & 4 & & \\
\hline Months since onset & & $54.4 \pm 25.4$ & & $68.8 \pm 30.7$ & NS \\
\hline Walking speed without an AFO (m/s) & & $0.6 \pm 0.1$ & & $0.6 \pm 0.1$ & NS \\
\hline
\end{tabular}

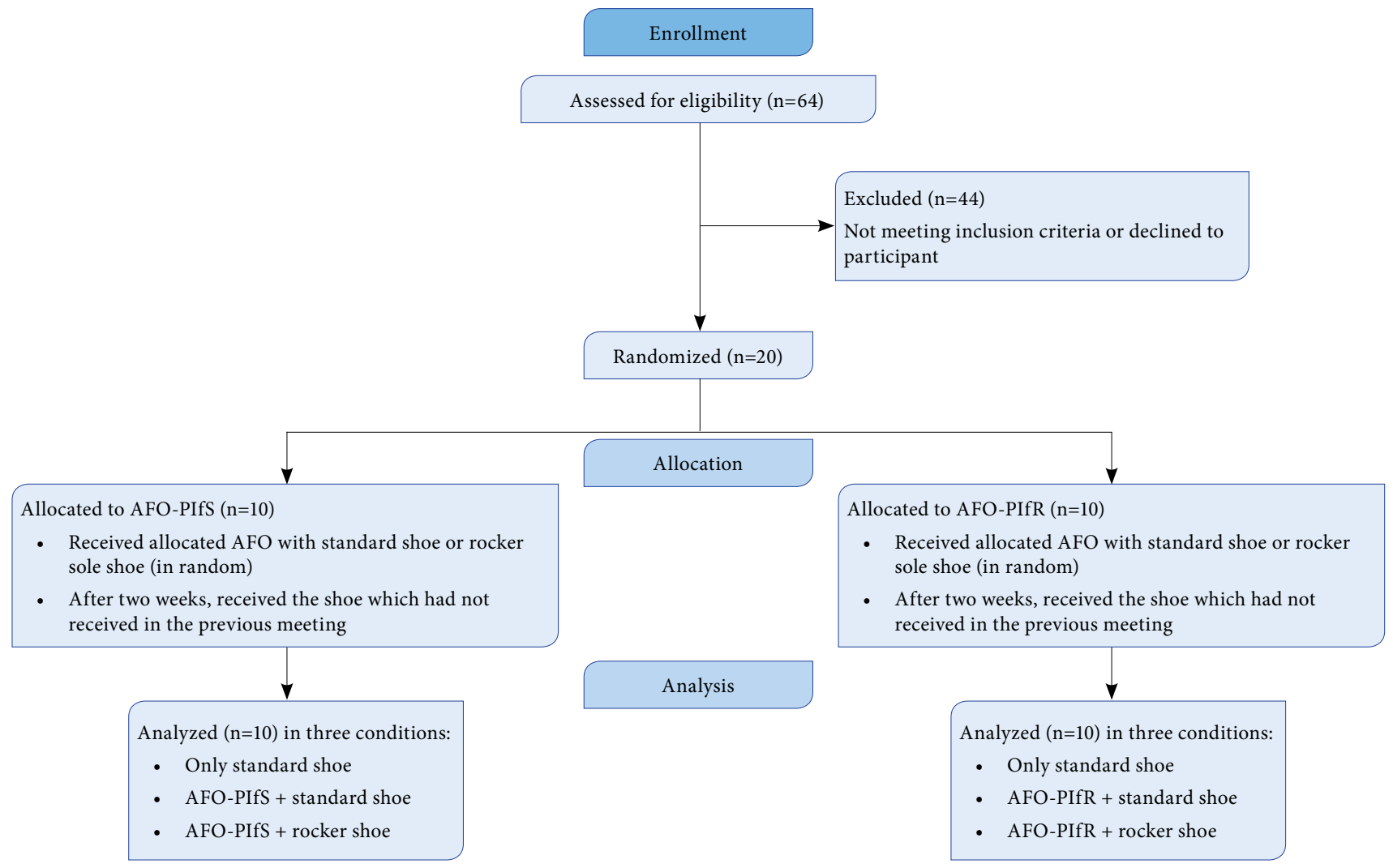

Figure 2. COSORT flow chart of the study.

AFO-PlfS: AFO with plantar flexion stop; AFO-PlfR: AFO with plantar flexion resistance; AFO: Ankle-foot orthosis. 


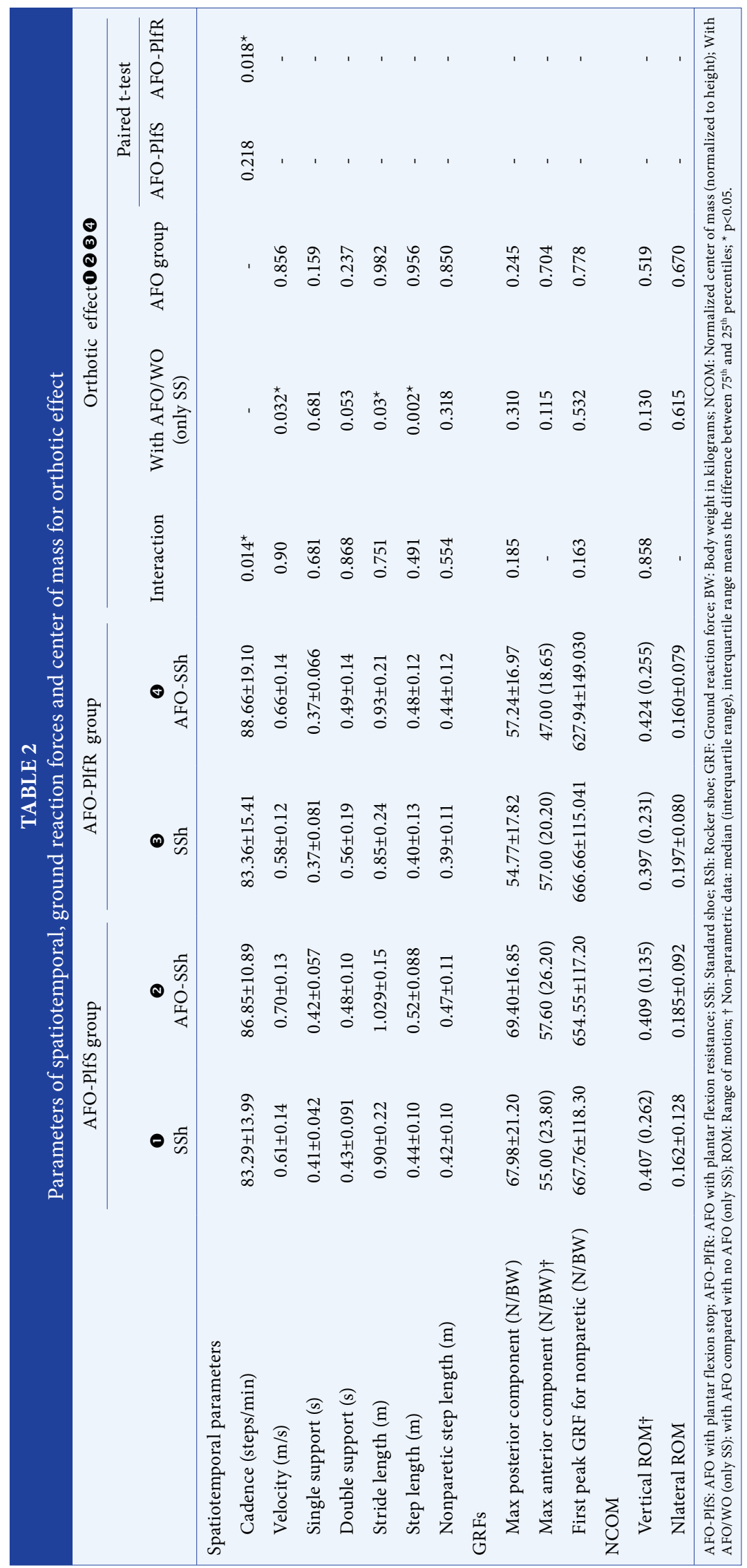




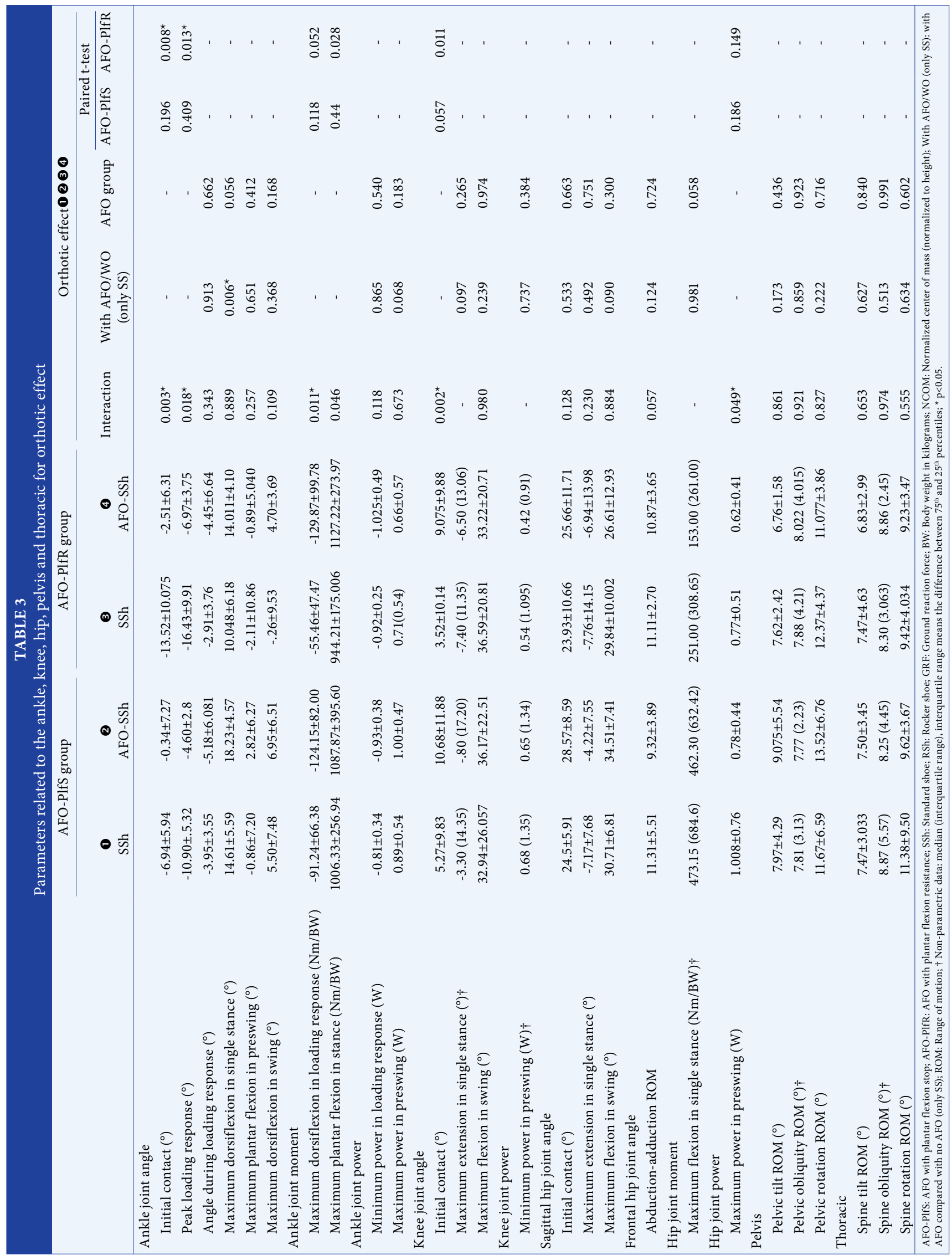




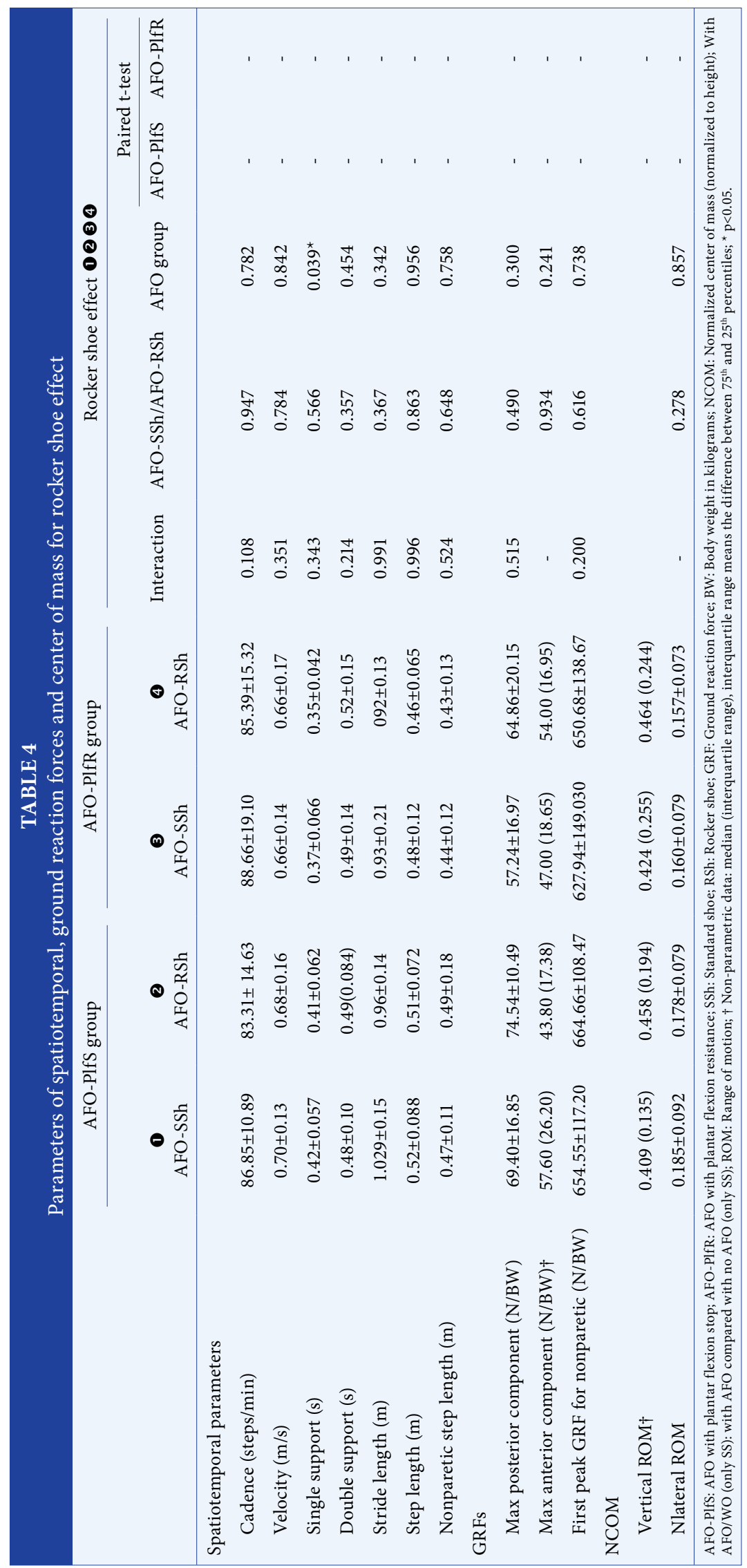




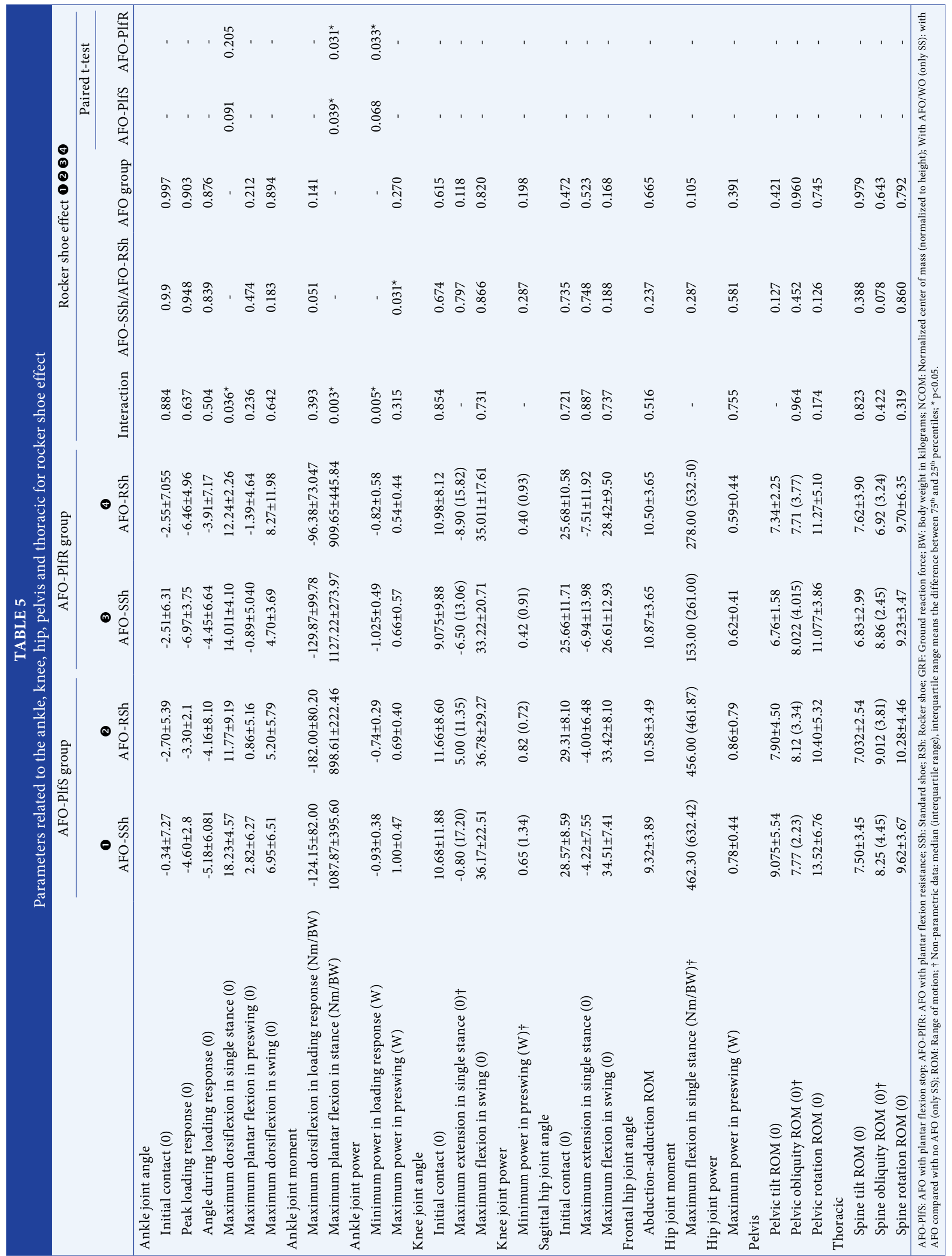


(a)

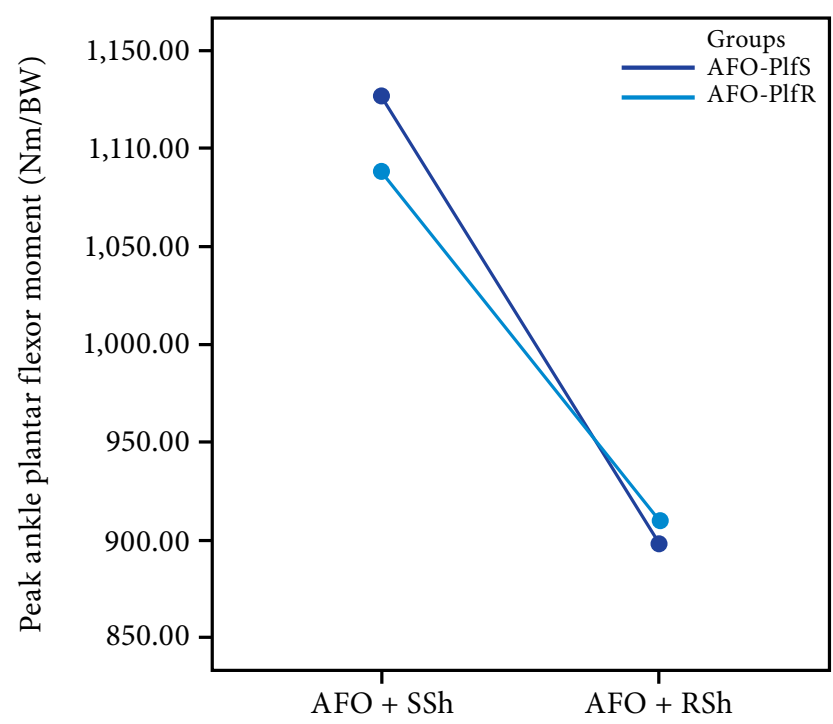

(b)

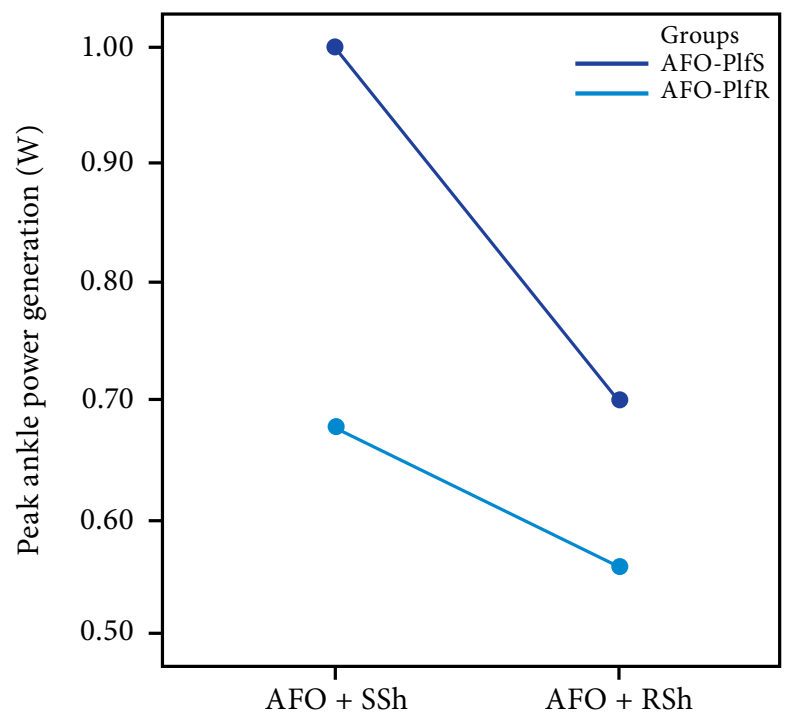

Figure 3. The significant changes of rocker shoe effect in ankle kinetics in two AFO group: (a) Peak ankle plantar flexor moment during preswing and (b) peak ankle power generation during preswing.

SSh: Standard shoe; RSh: Rocker shoe.

orthotic effect was determined by comparing gait without an AFO (only SSh) and SSh on an AFO in two AFO groups. The rocker shoe effect was also determined by comparing walking with an RSh on an AFO and walking with SSh on an AFO in two groups. Of a total of 38 gait indices, the mean $\pm S D$, median (IQR), and $p$ values are shown in Tables 2-5.

\section{Orthotic effect}

The repeated two-way ANOVA showed a significant main effect of only SSh and SSh on an AFO for some parameters in both AFO groups including walking speed (AFO-PlfS: 0.09\%, AFO-PlfR: $0.08 \%$ ), step length (AFO-PlfS: $0.08 \%$, AFO-PlfR: $0.08 \%$ ), stride length (AFO-PlfS: $0.12 \%$, AFO-PlfR: 0.08\%), maximum ankle dorsiflexion in single support (AFO-PlfS: 3.6\%, AFO-PlfR: $3.96 \%$ ), indicating that theses gait parameters were increased while walking with an AFO compared with no AFO $(\mathrm{p}<0.05)$. The significant interaction effects were found for cadence $(\mathrm{p}=0.014)$, ankle dorsiflexion angle at initial contact $(\mathrm{p}=0.003)$, peak ankle plantar flexion in loading response $(\mathrm{p}=0.018)$, peak dorsiflexion moment in loading response $(\mathrm{p}=0.011)$, knee flexion at initial contact $(\mathrm{p}=0.002)$, and peak plantar flexion moment in preswing $(p=0.046)$. Paired t-test analysis was conducted for parameters to compare two conditions in each group. For the AFO-PlfR group, all six parameters were significantly increased, while walking with the AFO, compared with no AFO, although these differences were not statistically significant for the AFO-PlfS group ( $\mathrm{p}>0.05)$.

Regarding the hip kinetic and kinematic, and 3D ROMs of pelvis and thorax during a gait cycle, no effects were found to be significant. In addition, there were no significant differences between the two AFO groups (effect of AFO type) concerning all gait variables $(\mathrm{p}>0.05)$.

\section{Rocker-shoe effect}

As shown in Table 5, a significant interaction was found in the peak plantar flexor ankle moment during preswing $(\mathrm{p}=0.003)$. Then, $\mathrm{t}$-test analysis revealed that the peak ankle moment was significantly reduced, while walking with an $\mathrm{AFO}$ wearing RSh, compared to AFO wearing SSh for both groups (AFO-PlfS: 228.61\%, AFO-PlfR: 178.21\%). Additionally, a main effect of SSh on an AFO and RSh on an AFO was found only in one item of gait parameters, the ankle power generation during preswing phase (Figure 3), showing a significant reduction, while walking with an AFO wearing RSh, compared to AFO wearing SSh for both groups (AFO-PlfS: $0.31 \%$, AFO-PlfR: $0.12 \%)$. These effects were not significant in other 
gait parameters $(p>0.05)$. Regarding the difference between two AFOs, finally, only single-support time showed a significant decrease in the AFO-PlfR group, compared to the AFO-PlfS group.

\section{DISCUSSION}

In the current study, we evaluated the effect of two AFOs, namely AFO-PlfS and AFO-PlfR, while wearing SSh and RSh. Our results proved the beneficial orthotic effect of both AFOs use on some spatiotemporal parameters and sagittal plane-ankle kinematics in the hemiplegic patients. The improvement in walking velocity, step length, and stride length was similar to previous works comparing gait without and with an AFO and, with the use of an AFO, the patients could achieve a more normalized gait due to the added stability in controlling ankle dorsiflexion. Our finding also revealed a increase for ankle angle at initial contact and in loading response, maximum dorsiflexion moment in loading response, and knee flexion at initial contact for both AFO group, although it reached statistical significance only in the AFO-PlfR group. It appears that the plantar flexion resistance function of the AFO can affect the first rocker more effectively during gait of stroke, supporting findings of previous studies concerning this function. ${ }^{[9,11,23]}$

Concerning the variables related to the third rocker for orthotic effect, only a significant increase of the ankle plantar flexion moment was found in the AFO-PlfR group which can be therapeutically beneficial in those suffering from central neurological disorders, such as stroke. With regard to the fact that the lower extremity in the paretic side of stroke can be accelerated forward via either the ankle plantar flexors during push-off or the hip flexors during pull-off, ${ }^{[21,24]}$ it has been assumed that pulling off the paretic limb using the hip flexors may compensate for the reduced push-off. ${ }^{[13,14,21]}$ One of the other compensatory mechanisms of push-off in the third rocker of paretic limb is also increased vertical GRF on the non-affected side corresponding to the first rocker of non-affected side. In the present study, we put forward the hypothesis of non-paretic-side GRF on this basis that individuals with lower limb disorders at push-off phase may increase negative work on use on non-affected leg such as vertical GRF, thereby, decreasing non-affected step length. ${ }^{[25,26]}$ We hypothesized that using an AFO, particularly AFO-PlfR, could decrease load on the proximal joint, such as hip joint, and negative work on the non-affected leg. Based on our results, the peak hip flexor moment and non-affected-side vertical GRFs were lower for the both groups, and the non-affected step length was higher than without them for both AFO groups, although these results did not reach statistical significance. On the other hand, although ankle moment improved in the AFO-PlfR group, it was not enough to demonstrate an improvement in the push-off function using an AFO, since other parameters related to the third rocker, including maximum anterior GRF, maximum ankle plantar flexion angle, maximum ankle power in preswing, were not improved. Moreover, we did not measure the angular velocity of ankle plantar flexion and the activity of the gastrocnemius and soleus muscles in the third rocker of gait. Our hypothesis regarding this study and the previous works is that using a passive AFO could not improve the third rocker function. ${ }^{[6]}$

In the present study, wearing both AFOs did not have significant effects on the hip kinetics and kinematics, as well as 3D ROM for pelvis and thorax compared with SSh. Pelvic and thoracic malalignment is gait characteristics of individuals with hemiplegia. ${ }^{[27]}$ Nevertheless, the most of orthotics studies have focused on more distal sites using an AFO due to more evident muscle weakness in these sites. Only a previous study reported that oil-damper AFO had an indirect effect on a better alignment of the thorax during the gait of subacute stroke. ${ }^{[28]}$ One possible explanation for our results could be that all gait assessments under no AFO and with AFO conditions were done during the same visit. Therefore, potential variability in the patients' walking, which may be observed in the gait data gaining on different sessions was not allowed. Moreover, individuals received gait training every day in the previous study, ${ }^{[28]}$ but our patients used AFOs just for the adaptation during daily activity. However, many studies have proven that gait training to stroke have a potential improvement in walking function, ${ }^{[29,30]}$ while limited studies have investigated the effect of using an orthosis on the stroke gait. Therefore it needs to additional studies whether different finding are obtained by training using and not using an AFO.

In our study, none of the gait parameters for the first and second rockers were significantly affected under RSh on AFOs compared with SSh on AFOs concerning the rocker-shoe effect. This finding seems to be reasonable, since the shape of both shoe rockers in heel and midfoot areas is almost the same. Concerning the third rocker, two items of parameters, 
peak ankle plantar flexor moment and peak ankle power generation, were significantly reduced while wearing RSh on AFOs compared to SSh on AFOs for both groups. These findings confirm the previous works that a rocker-sole footwear decreases need for the first metatarsophalangeal joint dorsiflexion ${ }^{[31]}$ during propulsion phase of walking, thereby, reducing joint sagittal motion of the forefoot ${ }^{[18]}$ and ankle, ${ }^{[18,32]}$ and decreasing the forefoot plantar pressures ${ }^{[32,33]}$ by reducing the Achilles tendon loading. For stroke survivors, since plantar flexor weakness could limit the maximal plantar flexion power and moment, ${ }^{[13]}$ we assumed that reduction of these parameters using an RSh might be considered just for improving rollover function of foot and facilitating weight progression during propulsive phase, while using an AFO with a rigid footplate, but not for a strong push-off.

While walking in RS on AFOs, walking speed, step length, kinematics and kinetics of more proximal joints to ankle, and non-affected GRF did not significantly change compared to walking with SSh on AFOs. Prior to study, we hypothesized that RSh could improve walking mobility and decrease compensatory strategies on more proximal joints to ankle and negative work on non-paretic limb. However, the lack of change in these indices may have a positive side, as well, since the use of RSh for stroke survivors does not have a negative effect, and patients can use an articulated AFO, improving the first and second rockers along with an RSh, facilitating rollover function during the third rocker of gait. It may be thought that RSh increases COM displacement due to it extra-height. However, the lateral and vertical COM displacement did not statistically change while wearing RSh on an AFO compared to wearing SSh on an AFO for both groups. A previous study showed that RSh utilizing along with a rigid AFO improved the functional mobility of post-stroke hemiplegia. ${ }^{[34]}$ Nevertheless, the effects of the RSh use on stroke balance still remain unclear and need further investigations.

As shown in Figure 3a, the reduction amount in the peak ankle moment is almost the same for both AFOs. The ankle power generation was also reduced for both AFO groups wearing RSh; however, but this reduction was greater in the AFO-PlfS group (Figure 3b). Thus, this further decrease could result from the decreased ankle angular velocity using AFO-PlfS and RSh. However, such a decrease in the ankle angular velocity is not considered beneficial in those with plantar flexor weakness, limiting the maximal plantar flexion power. ${ }^{[20]}$ On the other hand, the single-support time showed a significant decrease in the AFO-PlfR group compared to AFO-PlfS group while wearing RSh, which cannot be considered therapeutically beneficial for AFO-PlfR group, since this change may decrease weight bearing on the paretic side in single-support phase. We, therefore, assume that, although using the RSh may facilitate rollover function during the third rocker of gait to some extent, it should be prescribed cautiously in stroke patients and an AFO may be more appropriate to be used with the SSh than the forefoot RSh.

One of the limitations of the present study is that all gait measurements were done in a single session without a routine training. Therefore, the potential effects of AFOs on more proximal sites to ankle were unable to be fully achieved. In addition, muscle activity was not measured for a better understanding of RSh effect on gait of stroke survivors. Finally, the relatively low sample size recruited from a rehabilitation center precludes the generalizability of these findings.

In conclusion, based to the orthotic effect, an AFO-PlfR can create a better function in the improvement of parameters related to the first rocker, but not to the third rocker. Although a rocker shoe can facilitate rollover for weight progression in the third rocker of gait, it could not make a strong push-off function in stroke patients. The rocker shoe should be prescribed cautiously in stroke patients.

\section{Acknowledgements}

We would like to thank all the participants in the present study. We are grateful to the University of Social Welfare and Rehabilitation Sciences and the Jawad Mowafaghian, Research Centre of Intelligent Neuro-Rehabilitation Technologies for providing the equipment. Trial registration number of this study: IRCT20190625044003N1.

Declaration of conflicting interests

The authors declared no conflicts of interest with respect to the authorship and/or publication of this article.

Funding

This study was financially supported by the Iran National Science Foundation with the Grant No. 95849762.

\section{REFERENCES}

1. Perry J. Davids JR. Gait analysis: normal and pathological function. J Pediatr Orthop 1992;12:815.

2. Wong AM, Pei YC, Hong WH, Chung CY, Lau YC, Chen CP. Foot contact pattern analysis in hemiplegic stroke patients: an implication for neurologic status determination. Arch Phys Med Rehabil 2004;85:1625-30. 
3. Lin PY, Yang YR, Cheng SJ, Wang RY. The relation between ankle impairments and gait velocity and symmetry in people with stroke. Arch Phys Med Rehabil 2006;87:562-8.

4. Nikamp CD, Buurke JH, van der Palen J, Hermens HJ, Rietman JS. Early or delayed provision of an ankle-foot orthosis in patients with acute and subacute stroke: a randomized controlled trial. Clin Rehabil 2017;31:798808.

5. de Sèze MP, Bonhomme C, Daviet JC, Burguete E, Machat $H$, Rousseaux $M$, et al. Effect of early compensation of distal motor deficiency by the Chignon ankle-foot orthosis on gait in hemiplegic patients: a randomized pilot study. Clin Rehabil 2011;25:989-98.

6. Daryabor A, Arazpour M, Aminian G. Effect of different designs of ankle-foot orthoses on gait in patients with stroke: A systematic review. Gait Posture 2018;62:26879.

7. Alam M, Choudhury IA, Bin Mamat A. Mechanism and design analysis of articulated ankle foot orthoses for drop-foot. ScientificWorldJournal 2014;2014:867869.

8. Yamamoto S, Hagiwara A, Mizobe T, Yokoyama O, Yasui T. Gait improvement of hemiplegic patients using an ankle-foot orthosis with assistance of heel rocker function. Prosthet Orthot Int 2009;33:307-23.

9. Yamamoto S, Fuchi M, Yasui T. Change of rocker function in the gait of stroke patients using an ankle foot orthosis with an oil damper: immediate changes and the short-term effects. Prosthet Orthot Int 2011;35:350-9.

10. Nolan KJ, Yarossi M. Preservation of the first rocker is related to increases in gait speed in individuals with hemiplegia and AFO. Clin Biomech (Bristol, Avon) 2011;26:655-60.

11. Ohata K, Yasui T, Tsuboyama T, Ichihashi N. Effects of an ankle-foot orthosis with oil damper on muscle activity in adults after stroke. Gait Posture 2011;33:102-7.

12. Daryabor A, Yamamoto S, Orendurff M, Kobayashi T. Effect of types of ankle-foot orthoses on energy expenditure metrics during walking in individuals with stroke: a systematic review. Disabil Rehabil. 2020 [Epub ahead of print]

13. Nadeau S, Gravel D, Arsenault AB, Bourbonnais D. Plantarflexor weakness as a limiting factor of gait speed in stroke subjects and the compensating role of hip flexors. Clin Biomech (Bristol, Avon) 1999;14:125-35.

14 Winter DA, Olney SJ, Conrad J, White SC, Ounpuu S, Gage WR. Adaptability of motor patterns in pathological gait. In: Winters JM, Woo SLY, editors. Multiple Muscle Systems. New York: Springer-Verlag; 1990. p. 680-93.

15. Waters RL, Mulroy S. The energy expenditure of normal and pathologic gait. Gait Posture 1999;9:207-31.

16. Danielsson A, Sunnerhagen KS. Energy expenditure in stroke subjects walking with a carbon composite ankle foot orthosis. J Rehabil Med 2004;36:165-8.

17. Forghany S, Nester CJ, Richards B. The effect of rollover footwear on the rollover function of walking. J Foot Ankle Res 2013;6:24.

18. Wu WL, Rosenbaum D, Su FC. The effects of rocker sole and $\mathrm{SACH}$ heel on kinematics in gait. Med Eng Phys 2004;26:639-46.
19. Farmani F, Mohseni-Bandpei MA, Bahramizadeh M, Aminian G, Abdoli A, Sadeghi-Goghari M. The influence of rocker bar ankle foot orthosis on gait in patients with chronic hemiplegia. J Stroke Cerebrovasc Dis 2016;25:2078-82.

20. Olney SJ, Griffin MP, Monga TN, McBride ID. Work and power in gait of stroke patients. Arch Phys Med Rehabil 1991;72:309-14.

21. Olney SJ, Griffin MP, McBride ID. Temporal, kinematic, and kinetic variables related to gait speed in subjects with hemiplegia: a regression approach. Phys Ther 1994;74:872-85.

22. Daryabor A, Arazpour M, Aminian G, Baniasad M, Yamamoto S. Design and evaluation of an articulated ankle foot orthosis with plantarflexion resistance on the gait: a case series of 2 patients with hemiplegia. J Biomed Phys Eng 2020;10:119-28.

23. Kobayashi T, Singer ML, Orendurff MS, Gao F, Daly WK, Foreman KB. The effect of changing plantarflexion resistive moment of an articulated ankle-foot orthosis on ankle and knee joint angles and moments while walking in patients post stroke. Clin Biomech (Bristol, Avon) 2015;30:775-80.

24. Olney S, Jackson V, George S. Gait re-education guidelines for stroke patients with hemiplegia using mechanical energy and power analyses. Physiotherapy Can 1988.40:242-48.

25. Grumillier C, Beyaert C, Haldric MA, Martinet N, Andre JM. Compensatory mechanism involving the hip of the unaffected side during gait in unilateral trans-tibial amputees. Computer Methods in Biomechanics and Biomedical Engineering 2007;10( suppl 1):125-6.

26. Forner-Cordero I, Furtado F, Cervera-Deval J, FornerCordero A. Ground reaction force patterns during gait in patients with lower limb lymphedema. Acta Fisiatr 2016;23:201-7.

27. Verheyden G, Vereeck L, Truijen S, Troch M, Herregodts I, Lafosse C, et al. Trunk performance after stroke and the relationship with balance, gait and functional ability. Clin Rehabil 2006;20:451-8.

28. Yamamoto S, Tanaka S, Motojima N. Comparison of ankle-foot orthoses with plantar flexion stop and plantar flexion resistance in the gait of stroke patients: A randomized controlled trial. Prosthet Orthot Int 2018;42:544-53.

29. Eng JJ, Tang PF. Gait training strategies to optimize walking ability in people with stroke: a synthesis of the evidence. Expert Rev Neurother 2007;7:1417-36.

30. Schröder J, Truijen S, Van Criekinge T, Saeys W. Feasibility and effectiveness of repetitive gait training early after stroke: A systematic review and meta-analysis. J Rehabil Med 2019;51:78-88.

31. Lin SC, Chen CP, Tang SF, Wong AM, Hsieh JH, Chen WP. Changes in windlass effect in response to different shoe and insole designs during walking. Gait Posture 2013;37:235-41.

32. Forghany S, Nester CJ, Richards B, Hatton AL, Liu A. Rollover footwear affects lower limb biomechanics during walking. Gait Posture 2014;39:205-12. 
33. Stewart L, Gibson JN, Thomson CE. In-shoe pressure distribution in "unstable" (MBT) shoes and flat-bottomed training shoes: a comparative study. Gait Posture 2007;25:648-51.
34. Farmani F, Mohseni Bandpei MA, Bahramizadeh M, Aminian G, Nikoo MR, Sadeghi-Goghari M. The effect of different shoes on functional mobility and energy expenditure in post-stroke hemiplegic patients using anklefoot orthosis. Prosthet Orthot Int 2016;40:591-7. 